\section{(2) OPEN ACCESS}

\title{
Risk of newly developing visual field defect and neurodegeneration after pars plana vitrectomy for idiopathic epiretinal membrane
}

\author{
Kunihiko Akino, ${ }^{1}$ Norihiro Nagai, ${ }^{1,2}$ Kazuhiro Watanabe, ${ }^{1}$ Norimitsu Ban, ${ }^{1}$ \\ Toshihide Kurihara, ${ }^{1}$ Atsuro Uchida, ${ }^{1}$ Hajime Shinoda, ${ }^{1}$ Kazuo Tsubota, ${ }^{1}$ \\ Yoko Ozawa (D) 1,2,3,4
}

\begin{abstract}
- Supplemental material is published online only. To view please visit the journal online (http://dx.doi.org/10.1136/ bjophthalmol-2020-317478).

${ }^{1}$ Department of Ophthalmology, Keio University School of Medicine, Shinjuku-ku, Tokyo, Japan

${ }^{2}$ Laboratory of Retinal Cell Biology, Keio University School of Medicine, Shinjuku-ku, Tokyo, Japan

${ }^{3}$ Department of Ophthalmology, St. Luke's International Hospital, Chuo-ku, Tokyo, Japan

${ }^{4}$ St. Luke's International University, Chuo-ku, Tokyo, Japan
\end{abstract}

\section{Correspondence to}

Yoko Ozawa, Department of Ophthalmology, Keio University School of Medicine, Shinjuku-ku, Tokyo, Japan; ozawa@a5.keio.jp; ozaway@luke.ac.jp

$\mathrm{KA}$ and NN contributed equally

Received 16 July 2020 Revised 3 September 2020 Accepted 13 September 2020

\section{ABSTRACT}

Background/Aims Pars plana vitrectomy (PPV) is widely performed in patients with idiopathic epiretinal membrane (iERM) to improve vision. Postoperative visual field defects (VFDs) have been previously reported. However, whether they occur when using the most recent PPV system, and the frequency of VFDs as measured by standard automated perimetry, remain poorly documented and were examined in this study. Methods Data of 30 eyes (30 patients; mean age, 66.1 years; 15 men) who underwent PPV for iERM during February 2016-June 2019 and had preoperative and postoperative visual field measurements using standard automated perimetry (Humphrey visual field analyser 30-2 program) were retrospectively analysed. Eyes with diseases other than iERM, including moderate-to-severe cataract or preoperative VFDs were excluded.

Results VFD, defined by the Anderson and Patella's criteria, was found in $73.3 \%$ of the eyes 1 month after PPV. After age adjustment, internal limiting membrane (ILM) peeling was identified as a risk factor for postoperative VFD ( $p=0.035 ; 95 \% \mathrm{Cl} 1.173$ to 92.8 ). Postoperative VFD was frequently observed nasally $(86.4 \%, p=0.002)$, and on optical coherence tomography measurements, ganglion cell layer (GCL) thinning was found temporal to the fovea $(p=0.008)$. Thinning of the superior and inferior retinal nerve fibre layers and of the $\mathrm{GCL}$ temporal to the fovea were significant in eyes after ILM peeling (all $p<0.05$ ).

Conclusion ILM peeling may cause inner retinal degeneration and lead to the development of VFDs after PPV, which should be further examined.

\section{INTRODUCTION}

Idiopathic epiretinal membrane (iERM) is found in $4 \%$ of the adults aged over 40 years ${ }^{1-3}$; its incidence is rising in the current ageing society. ${ }^{1}$ Due to the advances in optical coherence tomography (OCT) and surgical instruments, pars plana vitrectomy (PPV) is widely applied for the treatment of iERM to improve quality of vision (QOV). Both central visual acuity, determined by foveal function, and the paracentral visual field, determined by retinal neural sensitivity surrounding the fovea, are implicated in achieving good QOV and treatment outcomes.

Better central visual acuity after PPV for iERM can be obtained in patients with better preoperative best- corrected visual acuity (BCVA) ${ }^{4}$ and/or less severe photoreceptor misalignment determined by the absence or lesser extent of a disrupted external limiting membrane and ellipsoid zone ${ }^{6-8}$ recorded by OCT. Additionally, population-based cohort analyses have shown that ERM is associated with a biomarker of glaucoma, that is, the defect of the retinal nerve fibre layer (RNFL), composed of retinal ganglion cell (RGC) axons, while it is not associated with another biomarker of glaucoma, that is, optic disc cupping. ${ }^{9-11}$ This suggests that iERM affects both the health condition of photoreceptor cells ${ }^{4-8}$ and RGCs and/or their axons. Thus, measuring the visual field would also be of value when evaluating the quality of the treatment outcome.

Several previous reports have shown large postoperative visual field defects (VFDs) after PPV for iERM. ${ }^{12-16}$ There are reports of large VFDs recorded by Goldmann perimetry being only observed in groups having undergone intravitreal injection of indocyanine green (ICG) during PPV for visualising the internal limiting membrane (ILM) for easier peeling, and not in groups where the ILM has been peeled without using ICG $^{12-14}$; thus, the toxicity of ICG, but not ILM peeling itself, was assumed to be the cause of large postoperative VFDs. Because ILM removal was found to reduce the recurrence of ERM after PPV from over 20\% to $2 \%,{ }^{17}$ many surgeons perform ILM peeling, although ICG may not be used. Recurrence of ERM and repeated surgery may be harmful to the retinal neurons, consuming time and resources, and increasing the mental burden. However, we still experience VFDs after PPV without using ICG. ${ }^{15}{ }^{18}$ Considering that the ILM is the basement membrane of the Müller glial cells, which contribute to the homoeostasis of the microenvironment, ${ }^{16}{ }^{19}$ its removal may affect Müller glial-cell function and/or surrounding neural tissue.

Under the recent trend of early glaucoma treatment, standard automated perimetry is basically used to sensitively detect VFDs. Thus, standard automated perimetry should also be used to evaluate VFDs as a surgical adverse event. Furthermore, currently, preperimetric glaucoma is diagnosed based on the inner retinal thickness measured using OCT. ${ }^{20}{ }^{21}$ Analyses of both total central retinal thickness (CRT) and postoperative neural thickness in each layer will help in sensitively evaluating neural loss.

Here, we analysed the data of patients who did not have VFDs before surgery to determine the incidence 
of postoperative VFDs recorded by standard automated perimetry and the change in retinal neural thickness in each layer to explore the pathogenesis of the VFDs. The impact of ILM peeling on the VFDs and neural thickness was also evaluated. The study results will help determine whether the surgical procedure should include processes such as ILM peeling by considering the risk-benefit balance in daily clinical practice.

\section{MATERIALS AND METHODS}

This retrospective study was performed at the Vitreo-Retina Surgical Division Clinic of the Department of Ophthalmology at Keio University Hospital. The procedures adhered to the tenets of the Declaration of Helsinki, and approval to perform this study was obtained from the Keio University School of Medicine Ethics Committee (approval number: 20100003).

\section{Patients}

The analysis was based on a detailed medical chart review. The study included 30 eyes of 30 patients who underwent PPV with or without ILM peeling for iERM between February 2016 and June 2019 in the Vitreo-Retina Surgical Division Clinic, Department of Ophthalmology, Keio University Hospital (Tokyo, Japan), and had no VFDs preoperatively. Patients who had conditions other than iERM, such as a secondary ERM, exhibited any preoperative VFDs with or without glaucoma, or had moderate-to-severe cataract, and underwent gas injection during PPV were excluded from the study. There were no eyes with preoperative RNFL defect.

\section{Eye examinations}

All patients underwent complete ophthalmological examinations, including BCVA measurement with a refraction test, slitlamp examination and binocular indirect ophthalmoscopy after pupil dilation with $0.5 \%$ tropicamide. Data at baseline (before PPV) and 1 month after PPV were collected.

\section{Visual field test}

The visual field was measured by standard automated perimetry, that is, the Humphrey visual field analyser (Carl Zeiss Meditec, Dublin, CA), using the central 30-2 (HFA 30-2) program with a Swedish interactive threshold algorithm. Data at baseline (before PPV) and 1 month after PPV were collected.

\section{Optical coherence tomography}

The OCT images were obtained by spectral-domain OCT (Heidelberg Spectralis OCT, Dossenheim, Germany). After pupil dilatation, the patients were asked to fixate on a target, and both horizontal and vertical images were recorded at baseline and after PPV using the 'repeat mode' based on the eye-tracking system. CRTwas defined as the distance between the inner retinal surface and the inner border of the retinal pigment epithelium (RPE), and central choroidal thickness (CCT) was defined as the distance between the hyper-reflective line corresponding to Bruch's membrane beneath the RPE and the inner surface of the sclera at the foveal centre. The thicknesses of each retinal layer were measured at the superior, inferior, temporal and nasal points to the fovea at a distance of $2000 \mu \mathrm{m}$. Measurements were performed using the calliper function of the OCT device, with manual adjustment, in the OCT system. Data at baseline (before PPV) and 1 month after PPV were collected.

\section{Surgery}

In all patients, PPV was performed by experienced surgeons (HS and $\mathrm{YO}$ ) for iERM removal with a 25 -gauge or 27-gauge system using forceps. ILM peeling was performed at the surgeon's discretion at the time of the surgery. The ILM was directly grasped and peeled with end-gripping forceps within an area of approximately $10^{\circ}$ eccentricity from the fovea. In 28 eyes, Brilliant Blue G (Merck KGaA, Darmstadt, Germany) was used at $1.0 \mathrm{mg} / \mathrm{mL}$ dissolved in BSS plus (Alcon Japan, Tokyo, Japan) (Keio University School of Medicine Ethics Committee; approval number: 20110115) to visualise the ILM, although some patients underwent staining only to confirm that the ILM was intact. Patients with mild cataract simultaneously underwent cataract surgery.

\section{Statistical analyses}

Data are expressed as mean $\pm S E$. Paired t-test, Mann-Whitney $\mathrm{U}$ test, $\chi^{2}$ test, and univariate and multivariate logistic regression analyses were performed using SPSS software (version 25.0, SPSS Japan, Tokyo, Japan). A p value $<0.05$ was considered statistically significant.

\section{RESULTS}

\section{Baseline characteristics and surgical outcome of iERM}

Of 30 eyes of 30 patients (mean age, $66.1 \pm 1.9$ (range, 43-84) years), 15 belonged to males (50\%) (online supplemental table 1). Mean BCVA was $0.202 \pm 0.034$ (range, -0.079 to 0.523 ) in LogMAR, mean CRT was 376 25 (range, 72-630) $\mu \mathrm{m}$ and mean CCT was $180 \pm 9$ (range, 110-294) $\mu \mathrm{m}$ at baseline. Nineteen eyes (63.3\%) underwent combined cataract surgery, and 17 eyes (56.7\%) underwent ILM peeling during PPV. Twenty-eight eyes $(93.3 \%)$ received Brilliant Blue G staining during surgery.

Overall, mean BCVA improved to $0.041 \pm 0.026(\mathrm{p}=0.0007)$ (online supplemental figure 1A) and CRT decreased to 283 $\pm 19 \mu \mathrm{m}(\mathrm{p}=0.0002)$ (online supplemental figure 1B) while CCT did not change $(188 \pm 9 \mu \mathrm{m} ; \mathrm{p}=0.512)$ at 1 month postoperatively (data not shown).

\section{Association between VFD and baseline findings}

VFD, defined by the Anderson and Patella's criteria (a cluster of three or more points in the pattern deviation plot within a single hemifield with $p<0.05$, one of which must have $p<0.01^{22}{ }^{23}$ ) appeared in 22 eyes (73.3\%) (online supplemental figure 2A). Among them, the defect spanned two quadrants in 9 eyes (40.9\%), three quadrants in 10 eyes (45.5\%) and four quadrants in 3 eyes (13.6\%) (online supplemental figure 2B). The nasal area was involved in 19 eyes (86.4\%), and the temporal area was involved in 9 eyes (40.9\%); the nasal area was involved significantly more frequently than the temporal area $(p=0.002)$ (online supplemental figure $2 \mathrm{C}$ ). The superior nasal quadrant was involved in 14 eyes (63.6\%), inferior nasal quadrant in 15 eyes (68.1\%), superior temporal quadrant in 6 eyes $(27.3 \%)$ and inferior temporal quadrant in 3 eyes (13.6\%) (online supplemen tal figure 2D).

Subsequently, we divided the eyes into two groups based on the presence or absence of newly developed postoperative VFD (table 1). The eyes with postoperative VFD had frequently undergone ILM peeling during PPV $(p=0.041)$. Moreover, the same result was obtained by multivariate logistic regression analysis after adjusting for age ( $\mathrm{p}=0.035 ; 95 \%$ CI 1.173 to 92.8$)$ (table 2).

\section{Thickness of retinal neural layers with or without VFD}

We compared the thickness of each retinal neural layer before and after PPV at a distance of $2000 \mu \mathrm{m}$ from the fovea. In contrast to the group with no postoperative VFD in which the ganglion cell layer (GCL) temporal to the fovea became thicker postoperatively (by $8.1 \pm 4.2 \mu \mathrm{m}$, paired t-test, $\mathrm{p}=0.009$, data not shown), the 
Table 1 Differences between eyes with or without postoperative visual field defect

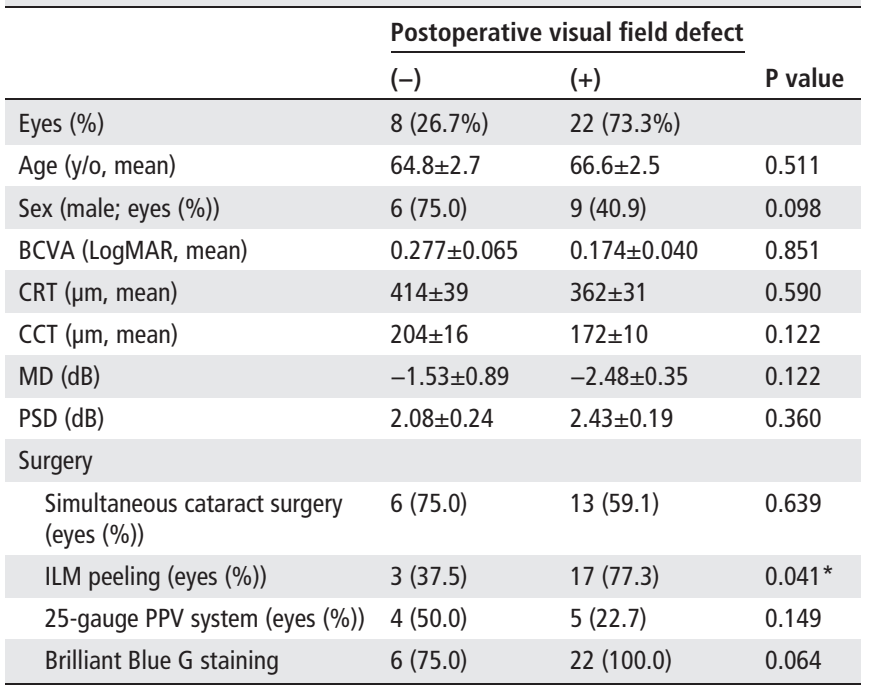

${ }^{*} p<0.05$.

Data are expressed as mean \pm SE. Mann-Whitney $\mathrm{U}$ test or $\chi^{2}$ test was performed.

BCVA, best-corrected visual acuity; CRT, central retinal thickness; CCT, central choroida

thickness; ILM, internal limiting membrane; LogMAR, logarithm of the minimum angle of

resolution; $\mathrm{MD}$, mean deviation; PSD, pattern SD; PPV, pars plana vitrectomy; y/o, years old.

Table 2 Impact of ILM peeling during pars plana vitrectomy on the presence of postoperative visual field defect

\begin{tabular}{llll}
\hline & OR & P value & $95 \% \mathrm{Cl}$ \\
\hline ILM peeling & 10.436 & $0.035^{*}$ & 1.173 to 92.8 \\
\hline
\end{tabular}

${ }^{*} \mathrm{p}<0.05$.

Multivariate logistic regression analysis after adjusting for age.

ILM, internal limiting membrane.

group with postoperative VFD showed thinning of the GCL at the same area 1 month after PPV compared with the baseline (by 10.7 $\pm 5.0 \mu \mathrm{m}$, paired t-test, $\mathrm{p}=0.045$, data not shown); the postoperative thinning (change) in GCL thickness temporal to the fovea was significantly severer in the groups with postoperative VFD $(p=0.031)$ (table 3$)$. There were no significant differences in the changes in outer retinal layer thickness (data not shown).

Thickness of retinal neural layers with or without ILM peeling Next, we divided the eyes based on whether ILM peeling was performed (17 eyes, $56.7 \%$ ) or not (13 eyes, 43.3\%) during PPV. Postoperative reduction in the thickness of the RNFL was significantly different in the presence or absence of ILM peeling, both

Table 3 Postoperative changes in GCL thickness in eyes with or without postoperative visual field defect

\begin{tabular}{lccl}
\hline & \multicolumn{2}{c}{ Postoperative visual field defect } & \\
\cline { 2 - 3 } GCL thickness $(\mu \mathrm{m})$ & \multicolumn{1}{c}{$(-)$} & $(+)$ & P value \\
\hline Superior & $4.6 \pm 3.8$ & $0.8 \pm 1.8$ & 0.656 \\
Inferior & $-0.1 \pm 2.9$ & $1.9 \pm 2.4$ & 0.410 \\
Temporal & $8.1 \pm 4.2$ & $-10.7 \pm 5.0$ & $0.031^{*}$ \\
Nasal & $3.5 \pm 2.0$ & $3.0 \pm 3.3$ & 0.925 \\
\hline
\end{tabular}

${ }^{*} \mathrm{p}<0.05,{ }^{* *} \mathrm{p}<0.01$.

Data are expressed as mean \pm SE. Mann-Whitney $U$ test was performed.

$\mathrm{GCL}$, ganglion cell layer. superior $(p=0.036)$ and inferior $(p=0.022)$ to the fovea (table 4$)$. Postoperative reduction in GCL thickness temporal to the fovea was also significantly greater in the group with ILM peeling $(p=0.023)$ (table 4). There were no differences between the preoperative and postoperative thicknesses of the RNFL and GCL at the other parts (data not shown).

Data on standard automated perimetry at 12 months were available for 14 of the 17 eyes with postoperative VFD after ILM peeling during PPV. The mean MD and PSD at 12 months were comparable to those at 1 month (1st month vs 12 th month; $\mathrm{MD},-4.35 \pm 0.65$ vs $-4.37 \pm 0.73, \mathrm{p}=0.969$; PSD, $7.61 \pm 0.97$ vs $7.38 \pm 0.88, \mathrm{p}=0.679$; both by paired t-test, data not shown), suggesting that the postoperative VFDs had not progressed by the 12 months, although further study is required.

A representative case with a VFD and thinning of the GCL temporal to the fovea after PPV with ILM peeling is shown in figure 1.

\section{DISCUSSION}

VFD, defined by the Anderson and Patella's criteria, appeared in 22 eyes (73.3\%) after PPV for iERM, although ICG was not used and the most recent PPV systems were used. ILM peeling was a significant risk factor for VFD development after adjusting for age. Several eyes had VFDs in multiple quadrants, and most frequently nasally, and GCL thinning was also frequently noted temporal to the fovea. Thinning of the superior and inferior RFNL and temporal GCL were significantly observed in the eyes that underwent ILM peeling during PPV.

VFD was found in $73 \%$ of the eyes in the current study using the central 30-2 (HFA 30-2) program of the standard automated perimetry, Humphrey visual field analyser. Previous reports have shown that large VFDs measured by Goldmann perimetry tests were found in $35 \%,{ }^{12} 57 \%{ }^{13}$ and $63 \%{ }^{14}$ of postoperative eyes with iERM, but only in eyes where ICG was used during ILM peeling. Conversely, VFDs determined by standard automated perimetry were found at a high frequency in postoperative eyes with iERM in this study, although ICG was not used, possibly because standard automated perimetry can detect slight defects and is more sensitive than the Goldmann perimetry tests. Another report using the Humphrey visual field analyser showed that postoperative mean visual field sensitivity in the eyes with iERM or macular hole treated with PPV decreased in the $10^{\circ}$ eccentricity (centre), but was preserved in eyes without glaucoma; moreover, the values of the remaining peripheral test points within $24^{\circ}$ improved postoperatively even in eyes that originally had glaucoma. ${ }^{15}$ The differences in the results could be because the previous study used the 24-2 program and evaluated the mean values of sensitivity, and this study used the 30-2 program; thus, wider analyses were performed, and VFDs were evaluated using the Anderson and Patella' criteria, according to which VFD was

Table 4 Postoperative changes in retinal thickness in the eyes with or without ILM peeling during pars plana vitrectomy

\begin{tabular}{lllll}
\hline & & \multicolumn{2}{l}{ ILM peeling } & \\
\cline { 3 - 4 } \multicolumn{2}{r}{ Retinal thickness $(\mu \mathrm{m})$} & $(-)$ & $(+)$ & P value \\
\hline RNFL & Superior & $0.6 \pm 5.7$ & $-18.6 \pm 6.6$ & $0.036^{*}$ \\
& Inferior & $0.5 \pm 4.0$ & $-18.3 \pm 6.6$ & $0.022^{*}$ \\
$\mathrm{GCL}$ & Temporal & $5.0 \pm 3.7$ & $-11.1 \pm 5.5$ & $0.023^{*}$ \\
\hline
\end{tabular}

${ }^{*} p<0.05$.

Data are expressed as mean \pm SE. Mann-Whitney $U$ test was performed.

$\mathrm{GCL}$, ganglion cell layer; ILM, internal limiting membrane; RNFL, retinal nerve fibre layer. 


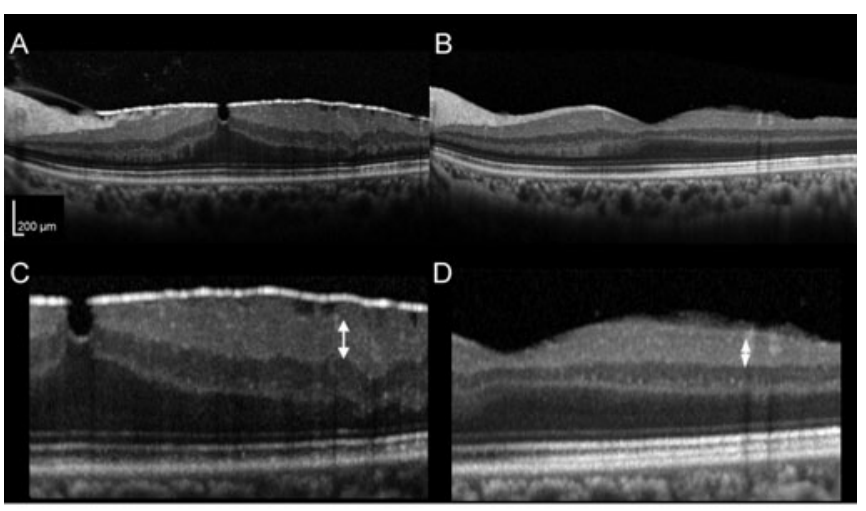

E

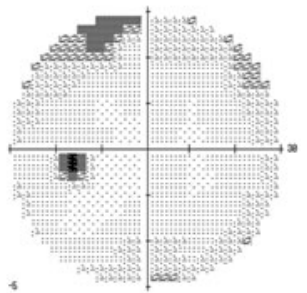

G

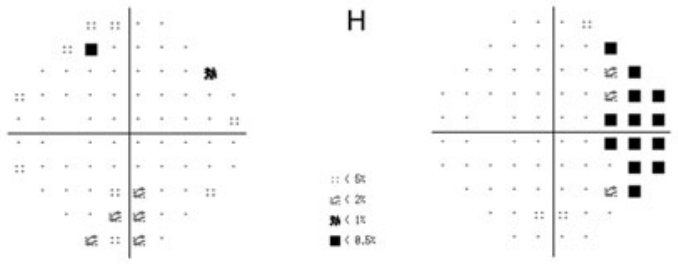

Figure 1 Preoperative and postoperative optical coherence tomography (OCT) images and visual field tests of a patient with postoperative visual field defect (VFD). Preoperative (A) and postoperative (B) OCT images of a patient with idiopathic epiretinal membrane who underwent pars plana vitrectomy with internal limiting membrane peeling. The respective preoperative and postoperative best-corrected visual acuities were 0.398 and -0.079 in LogMAR, and respective thicknesses of the ganglion cell layer at $2000 \mu \mathrm{m}$ temporal to the foveal centre were $72 \mu \mathrm{m}(C$, arrow) and $18 \mu \mathrm{m}(\mathrm{D}$, arrow). The greyscale $(E, F)$ and pattern deviation map $(G, H)$ analysed by the Humphrey visual field analyser showed nasal VFD after surgery $(F, H)$, which was not present preoperatively $(E, G)$.

defined when a local abnormality was detected. Considering these points, this study may have more sensitively detected local neural damage than all previous reports listed earlier.

ILM peeling was found to be a risk factor for postoperative VFD in this study. ILM is a basement membrane and a part of the Müller glial cells. Moreover, in the surgically peeled ILM, cellular components of Müller glial cells themselves were reported to be contaminated by electron microscopy. ${ }^{12}$ Considering that Müller glial cells have a critical role in the homoeostasis of the retinal neural microenvironment, ${ }^{24}$ abnormality or loss of Müller glial cells may easily cause retinal degeneration. This mechanism may also be involved in addition to the direct mechanical stress to the neurons at the time of peeling; one report showed that neural components were found in the pathological analysis of resected ERM, ${ }^{16}$ suggesting that retinal neurons could be incidentally removed during surgery, and the possibility could be theoretically increased with ILM peeling.

Although we did not use ICG to visualise the ILM, we used Brilliant Blue $G$, which could have affected the retinal neurons. However, most (93\%) eyes were injected with brilliant Blue G even when the ILM was not peeled; in these eyes, the injection was performed to confirm that the ILM was not peeled. Brilliant blue $G$ was reported to be safer than ICG in a neural cell line, ${ }^{25}$ and its intravitreal injection did not cause RGC reduction ${ }^{26}$ or visual function impairment as measured by electroretinography. ${ }^{26} 27$ The safety was also analysed by comparing it with ICG and trypan blue, by injecting Brilliant Blue $G$ into the subretinal space to find no apoptotic cells in the outer and inner layers of the retina and the RPE in animals. ${ }^{28}$ Therefore, Brilliant Blue G may have minimally influenced the appearance of VFDs in this study. Taken together with the fact that we did not use ICG, which was proven to be toxic for the retina, ${ }^{12-14}$ the VFDs observed in the current study were most likely due to the ILM peeling itself.

VFD was frequently found in the nasal part. Consistently, GCL thinning temporal to the fovea was greater in eyes with postoperative VFD than in those without. Additionally, VFDs spread to both the superior and inferior quadrants, which is not observed in typical glaucoma. ILM peeling decreased the thickness of the RNFL, both superior and inferior to the fovea, and the GCL temporal to the fovea, suggesting that ILM peeling may have damaged the RGCs at the temporal area of the fovea including the superior and inferior quadrants. However, whether cell bodies had been damaged first and axons were then affected or vice versa should be further analysed. Postoperative dissociated optic nerve fibre layer (DONFL), whose relationship with VFD remains controversial, ${ }^{29} 30$ was only observed in one eye, which had a postoperative VFD. However, this could be because DONFL was found to have clearly developed approximately 6 months postoperatively, and its relative absence 1 month postoperatively is reasonable. ${ }^{30}$

The ILM was directly grasped with forceps and slowly peeled, and the grasping point was determined in each eye; thus, it varied among the eyes. However, the RGCs of the temporal area to the fovea were the most affected. Thus, the damage was most likely unrelated to the mechanical damage at the time of ILM grasping. RGCs at the temporal area of the fovea involve more RGCs positive for calcium-binding proteins: parvalbumin, calbindin and calretinin, ${ }^{31}$ and these cells show distinct photopic responses when they are stressed by increased intraocular pressure in animal models, ${ }^{31}$ suggesting the possibility that RGCs temporal to the fovea may have different and vulnerable characteristics than the RGCs in other parts of the retina.

Alternatively, thinning of the GCL temporal to the fovea could be related to the displacement of the retina after ILM peeling towards the optic disc, as reported for macular hole ${ }^{32}$ and diabetic macular oedema, ${ }^{33}$ possibly because of the contraction and/or linearisation of the retinal tissue after removing the tension of the ILM to retain the retinal neurons arcuate, and the contractile force may have damaged the RGCs including their synapses and axons.

Not performing ILM peeling could increase ERM recurrences, ${ }^{17} 3435$ which would be disadvantageous. However, one should carefully consider whether to include ILM peeling to the procedure, considering that it may increase the risk of VFD. Particularly, if the eye shows signs of glaucoma, ILM peeling in the first operation for iERM may be avoided. Moreover, apart from the iERM clinic, at the time of screening glaucoma, we could ask patients who meet the Anderson and Patella's criteria in perimetry tests whether they have histories of PPV for iERM.

The limitations of this study were a relatively small sample, a retrospective design and the inclusion of patients whose eyes were injected with Brilliant Blue $G$ and patients whose eyes were not, although the last point may not have had a marked impact, as discussed earlier. ILM peeling was performed at the surgeon's discretion at the time of surgery, and this could have introduced 
bias. Further prospective studies would be required. Combined cataract surgery was performed in some of the patients; however, it may not cause VFDs.

In summary, ILM peeling was found to be a risk factor for developing VFDs after PPV for iERM. Postoperative GCL thinning was significant at the temporal part of the fovea, and VFDs frequently appeared at the nasal part. ILM peeling reduced the thickness of the GCL temporal to the fovea and of the RNFL both superior and inferior to the fovea. Although ILM peeling during iERM might have merit in preventing recurrences, ${ }^{17} 3435$ further study is warranted to reconsider the impact of ILM peeling for iERM on QOV.

Acknowledgements We appreciate all the colleagues including comedical staffs in Department of Ophthalmology, Keio University Hospital.

Contributors Conception and designs: NN, YO. Data collection: KA, NN, YO. Analysis and interpretation: KA, NN. Obtain funding: NN, KW, AU, TK, NB, HS. Review the manuscript: HS, KT. Overall responsibility: YO.

Funding The authors have not declared a specific grant for this research from any funding agency in the public, commercial or not-for-profit sectors.

Competing interests None declared.

Provenance and peer review Not commissioned; externally peer reviewed.

Data availability statement All data relevant to the study are included in the article or uploaded as supplemental information.

Supplemental material This content has been supplied by the author(s). It has not been vetted by BMJ Publishing Group Limited (BMJ) and may not have been peerreviewed. Any opinions or recommendations discussed are solely those of the author(s) and are not endorsed by BMJ. BMJ disclaims all liability and responsibility arising from any reliance placed on the content. Where the content includes any translated material, BMJ does not warrant the accuracy and reliability of the translations (including but not limited to local regulations, clinical guidelines, terminology, drug names and drug dosages), and is not responsible for any error and/ or omissions arising from translation and adaptation or otherwise.

Open access This is an open access article distributed in accordance with the Creative Commons Attribution Non Commercial (CC BY-NC 4.0) license, which permits others to distribute, remix, adapt, build upon this work non-commercially, and license their derivative works on different terms, provided the original work is properly cited, appropriate credit is given, any changes made indicated, and the use is noncommercial. See: http://creativecommons.org/licenses/by-nc/4.0/.

\section{ORCID iD}

Yoko Ozawa http://orcid.org/0000-0003-4797-5705

\section{REFERENCES}

1 Miyazaki M, Nakamura $\mathrm{H}$, Kubo M, et al. Prevalence and risk factors for epiretinal membranes in a Japanese population: the Hisayama study. Graefes Arch Clin Exp Ophthalmol 2003;241:642-6.

2 Kawasaki R, Wang JJ, Sato $\mathrm{H}$, et al. Prevalence and associations of epiretinal membranes in an adult japanese population: the Funagata study. Eye (Lond) 2009:23:1045-51.

$3 \mathrm{Ng} \mathrm{CH}$, Cheung N, Wang JJ, et al. Prevalence and risk factors for epiretinal membranes in a multi-ethnic United States population. Ophthalmology 2011;118:694-9.

4 Scheerlinck LM, van der Valk R, van Leeuwen R. Predictive factors for postoperative visual acuity in idiopathic epiretinal membrane: a systematic review. Acta Ophthalmol 2015:93:203-12.

5 Minami S, Shinoda $H$, Shigeno $Y$, et al. Effect of axial length and age on the visual outcome of patients with idiopathic epiretinal membrane after pars plana vitrectomy. Sci Rep 2019;9:19056.

6 Mitamura Y, Hirano K, Baba T, et al. Correlation of visual recovery with presence of photoreceptor inner/outer segment junction in optical coherence images after epiretinal membrane surgery. Br J Ophthalmol 2009:93:171-5.

7 Inoue M, Morita S, Watanabe Y, et al. Preoperative inner segment/outer segment junction in spectral-domain optical coherence tomography as a prognostic factor in epiretinal membrane surgery. Retina 2011;31:1366-72.
$8 \mathrm{Kim} \mathrm{JH}$, Kim YM, Chung EJ, et al. Structural and functional predictors of visual outcome of epiretinal membrane surgery. Am J Ophthalmol 2012;153:103-10. e101.

9 You Q, Xu L, Jonas JB. Prevalence and associations of epiretinal membranes in adult Chinese: the Beijing Eye Study. Eye (Lond) 2008:22:874-9.

10 McCarty DJ, Mukesh BN, Chikani V, et al. Prevalence and associations of epiretinal membranes in the visual impairment project. Am J Ophthalmol 2005;140:288-94.

11 Klein R, Klein BE, Wang Q, et al. The epidemiology of epiretinal membranes. Trans Am Ophthalmol Soc 1994;92:403-425; discussion 425-430.

12 Haritoglou C, Gandorfer A, Gass CA, et al. The effect of indocyanine-green on functional outcome of macular pucker surgery. Am J Ophthalmol 2003;135:328-37.

13 Uemura A, Kanda S, Sakamoto Y, et al. Visual field defects after uneventful vitrectomy for epiretinal membrane with indocyanine green-assisted internal limiting membrane peeling. Am J Ophthalmol 2003;136:252-7.

14 von Jagow B, Hoing A, Gandorfer A, et al. Functional outcome of indocyanine green-assisted macular surgery: 7-year follow-up. Retina 2009;29:1249-56.

15 Tsuchiya S, Higashide T, Sugiyama K. Visual field changes after vitrectomy with internal limiting membrane peeling for epiretinal membrane or macular hole in glaucomatous eyes. PLoS One 2017;12:e0177526.

$16 \mathrm{Kim} \mathrm{CY}$, Lee JH, Lee SJ, et al. Visual field defect caused by nerve fiber layer damage associated with an internal limiting lamina defect after uneventful epiretinal membrane surgery. Am J Ophthalmol 2002;133:569-71.

17 De Novelli FJ, Goldbaum M, Monteiro MLR, et al. Recurrence rate and need for reoperation after surgery with or without internal limiting membrane removal for the treatment of the epiretinal membrane. Int J Retina Vitreous 2017;3:48.

18 Uchida A, Shinoda K, Matsumoto CS, et al. Acute visual field defect following vitrectomy determined to originate from optic nerve by electrophysiological tests. Case Rep Ophthalmol 2012;3:396-405.

19 Terasaki H, Miyake Y, Nomura R, et al. Focal macular ERGs in eyes after removal of macular ILM during macular hole surgery. Invest Ophthalmol Vis Sci 2001;42:229-34

20 Nakano N, Hangai M, Nakanishi $\mathrm{H}$, et al. Macular ganglion cell layer imaging in preperimetric glaucoma with speckle noise-reduced spectral domain optical coherence tomography. Ophthalmology 2011;118:2414-26.

21 Chen TC, Hoguet A, Junk AK, et al. Spectral-domain OCT: helping the clinician diagnose glaucoma: a report by the American Academy of Ophthalmology. Ophthalmology 2018:125:1817-27.

22 Anderson DR, Patella VM. Automated static perimetry. 2nd edn edn. St. Louis: Mosby, 1999

23 Ono T, Yuki K, Asaoka R, et al. Glaucomatous visual field defect severity and the prevalence of motor vehicle collisions in Japanese: a hospital/clinic-based cross-sectional study. J Ophthalmol 2015;2015:497067.

24 Bringmann A, landiev I, Pannicke T, et al. Cellular signaling and factors involved in Muller cell gliosis: neuroprotective and detrimental effects. Prog Retin Eye Res 2009;28:423-51.

25 Boyd SR, Zachary I, Chakravarthy U, et al. Correlation of increased vascular endothelia growth factor with neovascularization and permeability in ischemic central vein occlusion. Arch Ophthalmol 2002;120:1644-50.

26 Remy M, Thaler S, Schumann RG, et al. An in vivo evaluation of Brilliant Blue $G$ in animals and humans. Br J Ophthalmol 2008:92:1142-7.

27 Enaida $\mathrm{H}$, Hisatomi T, Goto Y, et al. Preclinical investigation of internal limiting membrane staining and peeling using intravitreal Brilliant Blue G. Retina 2006:26:623-30

28 Ueno A, Hisatomi T, Enaida H, et al. Biocompatibility of Brilliant Blue $\mathrm{G}$ in a rat model of subretinal injection. Retina 2007:27:499-504.

29 Tadayoni R, Svorenova I, Erginay A, et al. Decreased retinal sensitivity after internal limiting membrane peeling for macular hole surgery. Br J Ophthalmol 2012;96:1513-16.

30 Ito Y, Terasaki H, Takahashi A, et al. Dissociated optic nerve fiber layer appearance after internal limiting membrane peeling for idiopathic macular holes. Ophthalmology 2005; 112:1415-20.

31 Tao X, Sabharwal J, Seilheimer RL, et al. Mild intraocular pressure elevation in mice reveals distinct retinal ganglion cell functional thresholds and pressure-dependent properties. J Neurosci 2019;39:1881-91.

32 Akahori T, Iwase T, Yamamoto K, et al. Macular displacement after vitrectomy in eyes with idiopathic macular hole determined by optical coherence tomography angiography. Am J Ophthalmol 2018;189:111-21.

33 Yoshikawa M, Murakami T, Nishijima K, et al. Macular migration toward the optic disc after inner limiting membrane peeling for diabetic macular edema. Invest Ophthalmol Vis Sci 2013;54:629-35.

34 Almony A, Nudleman E, Shah GK, et al. Techniques, rationale, and outcomes of internal limiting membrane peeling. Retina 2012;32:877-91.

35 Haritoglou C, Gass CA, Schaumberger M, et al. Macular changes after peeling of the internal limiting membrane in macular hole surgery. Am J Ophthalmol 2001;132:363-8. 